OR Spektrum (1996) 18: 97-105

\title{
Collinearity between the Shapley value and the egalitarian division rules for cooperative games
}

\author{
Irinel Dragan $^{1}$, Theo Driessen ${ }^{2}$, Yukihiko Funaki ${ }^{3}$ \\ 1 Department of Mathematics, University of Texas at Arlington, Arlington, TX 76019-0408, USA \\ 2 Department of Applied Mathematics, University of Twente, Enschede, The Netherlands \\ ${ }^{3}$ Faculty of Economics, Toyo University, Hakusan, Bunkyo-ku, Tokyo 112, Japan
}

Received: 27 September 1994/Accepted: 27 February 1995

\begin{abstract}
For each cooperative $n$-person game $v$ and each $h \in\{1,2, \ldots, n\}$, let $v_{h}$ be the average worth of coalitions of size $h$ and $v_{h}^{i}$ the average worth of coalitions of size $h$ which do not contain player $i \in N$. The paper introduces the notion of a proportional average worth game (or PAWgame), i.e., the zero-normalized game $v$ for which there exist numbers $c_{h} \in \mathbb{R}$ such that $v_{h}-v_{h}^{i}=c_{h}\left(v_{n-1}-v_{n-1}^{i}\right)$ for all $h \in\{2,3, \ldots, n-1\}$, and $i \in N$. The notion of average worth is used to prove a formula for the Shapley value of a PAWgame. It is shown that the Shapley value, the value representing the center of the imputation set, the egalitarian nonseparable contribution value and the egalitarian non-average contribution value of a PAW-game are collinear. The class of PAW-games contains strictly the class of $k$-coalitional games possessing the collinearity property discussed by Driessen and Funaki (1991). Finally, it is illustrated that the unanimity games and the landlord games are PAW-games.
\end{abstract}

Zusammenfassung. Sei $v$ ein kooperatives $n$-Personenspiel und sei $h \in\{1,2, \ldots, n\}$. Mit $v_{h}$ bezeichnen wir die mittlere Auszahlung aller Koalitionen der Größe $h$ und mit $v_{h}^{i}$ die mittlere Auszahlung aller Koalitionen der Größe $h$, die den Spieler $i \in N$ nicht enthalten. In dieser Arbeit, führen wir den Begriff des Spieles mit proportionaler mittlerer Auszahlung (oder PMA-Spiel) ein. Diese sind null-reduzierte Spiele $v$, für die Zahlen $c_{h} \in \mathbb{R}$ existieren, sodaß die Beziehung $v_{h}-v_{h}^{i}=c_{h}\left(v_{n-1}-v_{n-1}^{i}\right)$ für jedes $h \in\{2$, $3, \ldots, n-1\}$ und $i \in N$ gilt. Der Begriff der mittleren Auszahlung wird dann benutzt, um eine Formel für den Shapley-Wert der PMA-Spiele abzuleiten. Wir zeigen, daß der Shapley-Wert, und die durch das Zentrum der Imputationsmenge, die gleichmäßigen nicht-separablen Beiträge, bzw. gleichmäßigen nicht-gemittelten Beiträge definierten Werte der PMA-Spiele kollinear sind. Die Klasse aller PMA-Spiele enthält im strengen Sinne die Klasse aller $k$ Koalitionsspiele, die die Kollinearitätseigenschaft haben (Driessen und Funaki, 1991). Schließlich zeigen wir, daß

Correspondence to: T. Driessen die Einstimmigkeitsspiele und die Grundbesitzerspiele auch PMA-Spiele sind.

Key words: Cooperative game, PAW-game, $k$-coalitional game, Shapley value, egalitarian division rules

Schlüsselwörter: Kooperatives Spiel, PMA-Spiel, $k$-Koalitionsspiel, Shapley-Wert, Regeln gleichmäßiger Aufteilungen

\section{Introduction}

In the framework of cooperative game theory, many onepoint solution concepts called values have been proposed. For specific classes of cooperative games, some of the onepoint solutions possess interesting geometric relationships, for example three of the relevant one-point solutions are collinear, i.e., lie on the same line. Driessen and Funaki (1991) studied the collinearity between the Shapley value, the egalitarian non-separable contribution value (called ENSC-value) and the value representing the center of the imputation set (called CIS-value) for the class of cooperative games called the $k$-coalitional games. Here the collinearity of three values expresses that the corresponding one-point solutions for a certain type of a cooperative game lie on the same line.

In the present paper, we introduce a class of games called proportional average worth games (PAW-games), which strictly contains the subclass of those $k$-coalitional games for which the three above-mentioned values are collinear. An $n$-person game is said to be a proportional average worth game, if for any nontrivial coalition size, the differences of the so-called average worth per player are proportional to the differences of the average worth per player with respect to the coalition size $n-1$. The notion of average worth was used by Dragan (1992) to present an average per capita formula for the Shapley value. We show that in general, beside the Shapley value, the ENSC-value, the CIS-value, as well as the egalitarian non-average con- 
tribution value (called ENAC-value), recently introduced by Driessen and Funaki (1993a), can all be reformulated in terms of average worth with respect to particular coalition sizes, namely ( $n-1)$-person, 1-person, and ( $n-2)$-person coalitions, respectively. As a matter of fact, the notion of average worth is our main tool in establishing the collinearity of three of the four values on the class of PAWgames. The collinearity results concerning the $k$-coalitional games obtained by Driessen and Funaki (1991) are therefore reproved as special cases of the same properties for PAW-games.

The paper is organized as follows. Section 2 deals with the formal definitions of the four values in question, the notion of average worth and the reformulations of all four values in terms of average worth. Two examples show that there are games in which the Shapley value is collinear with the ENSC-value and CIS-value, but the Shapley value is not collinear with the ENAC-value and CIS-value, and games in which there is no collinearity at all. In Sect. 3, we introduce the PAW-games and prove that for such games the Shapley value is collinear with the ENSC-value and CIS-value and also with the ENAC-value and CISvalue. For four-person games the collinearity is even a sufficient condition for being PAW-games, a fact which is not true in general, as shown by a five-person game discussed in the previous section. The unanimity games are PAWgames, but the sum of two PAW-games is not in general a PAW-game. Finally, Section 4 is devoted to the class of $k$ coalitional $n$-person games. The relationship between $k$ coalitional games and PAW-games is elucidated in the sense that each $k$-coalitional game for which the collinearity property between the Shapley value, the ENSC-value and the CIS-value holds, belongs to the class of PAWgames. An example shows that the latter class is strictly larger. The paper ends with the study of the class of landlord games; it is illustrated that the landlord games are PAW-games, but not necessarily $k$-coalitional games.

\section{Notions and solution concepts}

Let $N$ be a finite set whose elements are called players. A cooperative or transferable utility game with player set $N$ is a real-valued function $v: 2^{N} \rightarrow \mathbb{R}$, on the set $2^{N}$ of all subsets of $N$, called coalitions. The worth $v(S)$ of coalition $S \subset N$ in the game $v$ represents the total profits that the members of $S$ can achieve due to their cooperative behaviour. It is a standard requirement that the empty coalition has no worth, i.e., $v(\emptyset):=0$. The number of players in a coalition $S$ is denoted by $|S|$. As usual, the players in the game are numbered in such a way that $N=\{1,2,3, \ldots, n\}$, where $n=|N|, n \geq 3$. The class of all cooperative $n$-person games is denoted by $G^{n}$.

The solution part of cooperative game theory focuses on the essential problem how to divide the overall profits $v(N)$ of the grand coalition among the players of the $n$-person game $v$. Four one-point solution concepts for cooperative games will be considered in this paper. The most well-known one-point solution concept has been introduced axiomatically by Shapley (1953). The classical for- mula for the Shapley value payoff to any player $i \in N$ in an $n$-person game $v$ is given by (cf. [13])

$$
S h_{i}(v)=\sum_{S \subset N ; i \in S}(n !)^{-1}(s-1) !(n-s) ![v(S)-v(S \backslash\{i\})]
$$

where $s=|S|$ for any $S \subset N$. As the Shapley value has many nice properties, making it perhaps the most popular solution, its computation may be regarded as a central problem. All algorithms (cf. [8], [10]) have the complexities $O\left(n \cdot 2^{n}\right)$, which makes the computation of the Shapley value tough for large $n$. For the purpose of simplification of computation, we compare the Shapley value concept with three easier computable solution concepts. The three one-point solution concepts have similar interpretations in the sense that the remaining overall profits are divided equally, given that each player is already paid some specified individual contribution. The individual contribution of any player $i \in N$ in an $n$-person game $v$ may be determined in one of the next three ways:

1. player $i$ 's individual worth $v(\{i\})$;

2. player $i$ 's separable (or marginal) contribution from an $(n-1)$-person coalition to the grand coalition, which is given by

$S C_{i}(v):=v(N)-v(M\{i\})$

3 . player $i$ 's average contribution from (n-2)-person coalitions to the grand coalition, which is given by

$$
A C_{i}(v):=v(N)-(n-2)^{-1} \sum_{j \in N \backslash\{i\}} v(N \backslash\{i, j\}) .
$$

For an interpretation of the expression in the right hand side of (2.3) as some average of marginal contributions of pairs of players including the relevant player $i$, we refer to Driessen and Funaki (1993a).

Subsequently, the egalitarian division of the surplus of the overall profits gives rise to three one-point solution concepts of the same kind, the value representing the center of the imputation set (CIS-value), the egalitarian nonseparable contribution value (ENSC-value), and the egalitarian non-average contribution value (ENAC-value), respectively. The CIS-value of an $n$-person game $v$ is formally defined by

$$
C I S_{i}(v):=v(\{i\})+n^{-1}\left[v(N)-\sum_{j \in N} v(\{j\})\right]
$$

for all $i \in N$; obviously, this value represents the center of the imputation set of the game $v$ given by

$I(v):=\left\{x \in \mathbb{R}^{n} \mid \sum_{j \in N} x_{j}=v(N)\right.$ and $x_{i} \geq v(\{i\})$ for all $\left.i \in N\right\}$.

Similarly, the ENSC-value and the ENAC-value are formally defined by

$$
\begin{aligned}
& E N S C_{i}(v):=S C_{i}(v)+n^{-1}\left[v(N)-\sum_{j \in N} S C_{j}(v)\right], \\
& E N A C_{i}(v):=A C_{i}(v)+n^{-1}\left[v(N)-\sum_{j \in N} A C_{j}(v)\right]
\end{aligned}
$$


for all $i \in N$. Note that the computation of the CIS-value, the ENSC-value and the ENAC-value requires beside the worth of the grand coalition, only the data of the 1-person, $(n-1)$-person and (n-2)-person coalitions, respectively. The next theorem states important results for the four values, that will be used throughout the paper; they will be expressed in terms of so-called average worth and the new formulas will replace (2.1)-(2.6).

Definition 2.1. Let $v \in G^{n}$, where $n \geq 3$.

(i) For each $h \in\{1,2, \ldots, n\}$, let $\Gamma_{h}:=\{S|S \subset N| S \mid,=h\}$ be the set of all coalitions of size $h$, and define the average worth of coalitions of size $h$ by

$v_{h}:=\left(\begin{array}{l}n \\ h\end{array}\right)^{-1} \sum_{S \in \Gamma_{h}} v(S)$

(ii) For each $h \in\{1,2, \ldots, n-1\}$ and each $i \in N$, let $\Gamma_{h}^{i}:=\{S|S \subset N| S \mid,=h, i \notin S\}$ be the set of all coalitions of size $h$ not containing player $i$, and define the average worth of coalitions of size $h$ not containing player $i$ by

$v_{h}^{i}:=\left(\begin{array}{c}n-1 \\ h\end{array}\right)^{-1} \sum_{S \in \Gamma_{h}^{i}} v(S)$.

For convenience, put $v_{n}^{i}:=0$ for all $i \in N$.

Theorem 2.2. Let $v \in G^{n}, n \geq 3$, and $i \in N$. We have

(i) $S h_{i}(v)=\sum_{h=1}^{n} h^{-1}\left(v_{h}-v_{h}^{i}\right)$

(ii) $\operatorname{CIS}_{i}(v)=n^{-1} v_{n}+(n-1)\left(v_{1}-v_{1}^{i}\right)$

(iii) $\operatorname{ENSC}_{i}(v)=n^{-1} v_{n}+\left(v_{n-1}-v_{n-1}^{i}\right)$

(iv) $\operatorname{ENAC}_{i}(v)=n^{-1} v_{n}+(n-2)^{-1}(n-1)\left(v_{n-2}-v_{n-2}^{i}\right)$.

Proof. (i) Distinguishing coalitions with different sizes and coalitions containing player $i$ or not, we deduce from the classical formula (2.1) for the Shapley value that

$$
\begin{aligned}
S h_{i}(v)= & \sum_{h=1}^{n} \sum_{S \in \Gamma_{h} ; i \in S} h^{-1}\left(\begin{array}{l}
n \\
h
\end{array}\right)^{-1}[v(S)-v(S \backslash\{i\})] \\
= & \sum_{h=1}^{n} h^{-1}\left(\begin{array}{l}
n \\
h
\end{array}\right)^{-1} \sum_{S \in \Gamma_{h} ; i \in S} v(S) \\
& -\sum_{h=2}^{n} h^{-1}\left(\begin{array}{l}
n \\
h
\end{array}\right)^{-1} \sum_{S \in \Gamma_{h} ; i \in S} v(S \backslash\{i\}) \\
= & \sum_{h=1}^{n} h^{-1}\left(\begin{array}{l}
n \\
h
\end{array}\right)^{-1}\left[\sum_{S \in \Gamma_{h}} v(S)-\sum_{S \in \Gamma_{h}^{i}} v(S)\right] \\
& -\sum_{h=2}^{n} h^{-1}\left(\begin{array}{l}
n \\
h
\end{array}\right)^{-1} \sum_{T \in \Gamma_{h-1}^{i}} v(T) \\
= & \sum_{h=1}^{n} h^{-1}\left(\begin{array}{l}
n \\
h
\end{array}\right)^{-1} \sum_{S \in \Gamma_{h}} v(S)-\sum_{h=1}^{n} h^{-1}\left(\begin{array}{l}
n \\
h
\end{array}\right)^{-1} \sum_{S \in \Gamma_{h}^{i}} v(S) \\
& -\sum_{m=1}^{n-1}(m+1)^{-1}\left(\begin{array}{c}
n \\
m+1
\end{array}\right)^{-1} \sum_{T \in \Gamma_{m}^{i}} v(T) .
\end{aligned}
$$

Substituting (2.7) and (2.8) respectively, we obtain

$$
\begin{aligned}
S h_{i}(v)= & \sum_{h=1}^{n} h^{-1} v_{h} \\
& -\sum_{h=1}^{n-1}\left[h^{-1}\left(\begin{array}{l}
n \\
h
\end{array}\right)^{-1}+(h+1)^{-1}\left(\begin{array}{c}
n \\
h+1
\end{array}\right)^{-1}\right] \cdot \sum_{S \in \Gamma_{h}^{i}} v(S) \\
= & \sum_{h=1}^{n} h^{-1} v_{h}-\sum_{h=1}^{n-1} h^{-1}\left(\begin{array}{c}
n-1 \\
h
\end{array}\right)^{-1} \sum_{S \in \Gamma_{h}^{i}} v(S) \\
= & \sum_{h=1}^{n} h^{-1} v_{h}-\sum_{h=1}^{n-1} h^{-1} v_{h}^{i}=\sum_{h=1}^{n} h^{-1}\left(v_{h}-v_{h}^{i}\right) .
\end{aligned}
$$

This proves part (i).

(ii) The proof of this part is left to the reader.

(iii) From (2.5), (2.2) and (2.7), (2.8) applied to $h=n-1$, we derive

$$
\begin{aligned}
\operatorname{ENSC}_{i}(v)= & n^{-1} v(N)+v(N)-v(N \backslash\{i\}) \\
& -n^{-1} \sum_{j \in N}[v(N)-v(N \backslash\{j\})] \\
= & n^{-1} v(N)+n^{-1} \sum_{j \in N} v(N \backslash\{j\})-v(N \backslash\{i\}) \\
= & n^{-1} v_{n}+v_{n-1}-v_{n-1}^{i} .
\end{aligned}
$$

(iv) From (2.6), (2.3) and (2.7), (2.8) applied to $h=n-2$, we derive

$$
\begin{aligned}
\operatorname{ENAC}_{i}(v)= & n^{-1} v(N)+v(N)-(n-2)^{-1} \sum_{k \in N \backslash\{i\}} v(N \backslash\{i, k\}) \\
& -n^{-1} \sum_{j \in N}\left[v(N)-(n-2)^{-1} \sum_{k \in N \backslash\{j\}} v(N \backslash\{j, k\})\right] \\
= & n^{-1} v(N)-(n-2)^{-1} \sum_{S \in \Gamma_{n-2}^{i}} v(S) \\
& +n^{-1}(n-2)^{-1} \sum_{j \in N} \sum_{k \in N \backslash\{j\}} v(N \backslash\{j, k\}) \\
= & n^{-1} v_{n}-(n-2)^{-1}(n-1) v_{n-2}^{i} \\
& +2 n^{-1}(n-2)^{-1} \sum_{S \in \Gamma_{n-2}} v(S) \\
= & n^{-1} v_{n}-(n-2)^{-1}(n-1) v_{n-2}^{i} \\
& +(n-2)^{-1}(n-1) v_{n-2} \\
= & n^{-1} v_{n}+(n-2)^{-1}(n-1)\left(v_{n-2}-v_{n-2}^{i}\right)
\end{aligned}
$$

The form (2.9) of the Shapley value formula has been introduced and proved by Dragan (1992). Because this average per capita formula for the Shapley value is our most important tool in this paper, we gave its proof here. A similar, but different average per capita formula for the Shapley value was used by Peleg (1992) in some game theoretic approach to voting theory by count and account. The ENSC-value and the CIS-value are well known concepts in the game theoretic literature (cf. [2], [3], [4], [6], [7], [9], [11], [14]). The ENAC-value has been introduced by Driessen and Funaki (1993a), who presented three motivations for the study of this value. The formula (2.12) for the ENAC-value, which is the sum of the egalitarian division of the overall profits and some part of the difference between two average worth with respect to (n-2)-person 
coalitions, provides us another motivation to concentrate on the ENAC-value, next to the ENSC-value and the CISvalue.

The main result of Theorem 2.2 that all four values can be expressed in terms of the average worth $v_{h}$ and $v_{h}^{i}$, $1 \leq h \leq n, i \in N$, suggests us to investigate the collinearity of three of the four values. In the next sections, we shall provide two types of sufficient conditions on the game so that the one-point solutions according to the Shapley value, the ENSC-value (or ENAC-value) and the CIS-value are collinear (i.e., lie on the same line). The importance of the collinearity property of three values (including the Shapley value) may be twofold. On the one hand the collinearity property may simplify (to some extent) the computation of the Shapley value (its simplification of computation, however, depends strongly on the complexity of the verification of the underlying condition guaranteeing the collinearity property of values), on the other the collinearity property is interesting on its own for the sake of comparison of values (e.g., to conclude which of the three onepoint solutions is the best or the worst for any of the players).

In the context of collinearity properties of several values, we may restrict ourselves, without loss of generality, to the class $G_{0}^{n}$ of zero-normalized n-person games defined by $G_{0}^{n}:=\left\{v \in G^{n} \mid v(\{i\})=0\right.$ for all $\left.i \in N\right\}$. Indeed, the process of zero-normalization transforms an arbitrary $n$-person game $v$ into the corresponding zero-normalized game $w \in G^{n}$ given by

$w(S):=v(S)-\sum_{j \in S} v(\{j\}) \quad$ for all $S \subset N$,

and it is straightforward to verify that each of the four values behaves nicely, i.e.,

$\sigma(w)=\sigma(v)-(v(\{1\}), v(\{2\}), \ldots, v(\{n\}))$

for any value $\sigma \in\{S h, E N S C, E N A C, C I S\}$. Therefore, the collinearity properties will not be affected by zero-normalization. Henceforward, we shall frequently use the results listed in the next corollary.

Corollary 2.3. Let $v \in G_{0}^{n}, n \geq 3$, and $i \in N$. We have

(i) $S h_{i}(v)=\sum_{h=2}^{n} h^{-1}\left(v_{h}-v_{h}^{i}\right)$

(ii) $\operatorname{CIS}_{i}(v)=n^{-1} v_{n}$

(iii) $\operatorname{ENSC}_{i}(v)-\operatorname{CIS}_{i}(v)=v_{n-1}-v_{n-1}^{i}$

(iv) $\operatorname{ENAC}_{i}(v)-\operatorname{CIS}_{i}(v)=(n-2)^{-1}(n-1)\left(v_{n-2}-v_{n-2}^{i}\right)$.

We conclude this section with two examples which will illustrate the lack of collinearity. Notice that for $v \in G_{0}^{3}$, we have always

$S h(v)=\frac{1}{2} \operatorname{ENSC}(v)+\frac{1}{2} \operatorname{CIS}(v)$ and $\operatorname{ENAC}(v)=\operatorname{CIS}(v)$,

so that we should consider games with at least four players to show the lack of collinearity.

Example 2.4. Consider the zero-normalized 4-person game $v$ given by $v(\{1,2\})=v(\{3,4\})=0, v(\{1,3\})=v(\{2,3\})=1$,

$v(\{1,4\})=v(\{2,4\})=2, v(\{1,2,3\})=1$,

$v(\{1,2,4\})=v(\{1,3,4\})=v(\{2,3,4\})=2, v(N)=3$.

Straightforward calculations yield all average worth as well as all differences of average worth:

$v_{2}=1, v_{2}^{1}=v_{2}^{2}=1, v_{2}^{3}=\frac{4}{3}, v_{2}^{4}=\frac{2}{3}$,

$v_{2}-v_{2}^{i}=0,0, \frac{-1}{3}, \frac{1}{3}$,

$v_{3}=\frac{7}{4}, v_{3}^{1}=v_{3}^{2}=v_{3}^{3}=2, v_{3}^{4}=1$,

$v_{3}-v_{3}^{i}=\frac{-1}{4}, \frac{-1}{4}, \frac{-1}{4}, \frac{3}{4}$,

$v_{4}=3$, for $i=1,2,3,4$, respectively.

From this and the new formulas (2.13)-(2.16), we get

$\operatorname{CIS}(v)=\frac{3}{4}(1,1,1,1), \operatorname{ENSC}(v)=\frac{1}{2}(1,1,1,3)$,

$S h(v)=\frac{1}{6}(4,4,3,7), \operatorname{ENAC}(v)=\frac{1}{4}(3,3,1,5)$.

We can check that there is no collinearity between the Shapley value and the $E N S C$-value (or $E N A C$-value) and the $C I S$-value; an exact explanation of the failure of such collinearities will be provided in Sect. 4. Note that the four values are coplanar, because we have $S h(v)=\frac{1}{3}[E N A C$ $(v)+E N S C(v)+C r S(v)]$. In the next example we shall have only one of the two collinearities.

Example 2.5. Consider the zero-normalized 5-person game $v$ given by

$v(\{4,5\})=1, v(S)=0$ for all $S \in \Gamma_{2}, S \neq\{4,5\}$,

$v(\{1,2,3\})=2, v(S)=1$ for all $S \in \Gamma_{3}, S \neq\{1,2,3\}$,

$v(\{1,2,3,4\})=4, v(\{1,2,3,5\})=5, v(\{1,2,4,5\})=6$,

$v(\{1,3,4,5\})=7, v(\{2,3,4,5\})=8, v(N)=10$.

Straightforward calculations yield all average worth as well as all differences of average worth:

$$
\begin{aligned}
& v_{2}=\frac{1}{10}, v_{2}^{1}=v_{2}^{2}=v_{2}^{3}=\frac{1}{6}, v_{2}^{4}=v_{2}^{5}=0, \\
& v_{2}-v_{2}^{i}=\frac{-1}{15}, \frac{-1}{15}, \frac{-1}{15}, \frac{1}{10}, \frac{1}{10}, \\
& v_{3}=\frac{11}{10}, v_{3}^{1}=v_{3}^{2}=v_{3}^{3}=1, v_{3}^{4}=v_{3}^{5}=\frac{5}{4}, \\
& v_{3}-v_{3}^{i}=\frac{1}{10}, \frac{1}{10}, \frac{1}{10}, \frac{-3}{20}, \frac{-3}{20}, \\
& v_{4}=6, v_{4}^{1}=8, v_{4}^{2}=7, v_{4}^{3}=6, v_{4}^{4}=5, v_{4}^{5}=4, \\
& v_{4}-v_{4}^{i}=-2,-1,0,1,2, \\
& v_{5}=10, \text { for } i=1,2,3,4,5, \text { respectively. }
\end{aligned}
$$

From this and the new formulas (2.13)-(2.16), we get

$\operatorname{CIS}(v)=(2,2,2,2,2), \operatorname{ENSC}(v)=(0,1,2,3,4)$,

$S h(v)=\frac{1}{4}(6,7,8,9,10), \operatorname{ENAC}(v)=\frac{1}{15}(32,32,32,27,27)$.

We discover easily that the Shapley value, the $E N S C$-value and the $C I S$-value lie on the same line, because $S h(v)=$ $\frac{1}{4} E N S C(v)+\frac{3}{4} C I S(v)$; instead the ENAC-value does not lie on the same line.

\section{Collinearity of values on the class of PAW-games}

Definition 3.1. Let $n \geq 3$. A game $v \in G_{0}^{n}$ is called a proportional average worth game (or PAW-game) if for all 
$h \in\{2,3, \ldots, n-1\}$ there exist numbers $c_{h} \in \mathbb{R}$ such that

$v_{h}-v_{h}^{i}=c_{h}\left(v_{n-1}-v_{n-1}^{i}\right) \quad$ for all $i \in N$.

Conditions (3.1) express that for any nontrivial coalition size, the differences of the average worth per player are proportional to the differences of such averages for the coalition size $n-1$.

Clearly, each zero-normalized 3-person game is a PAWgame, because (3.1) holds with $c_{2}=1$. Further, each zeronormalized symmetric $n$-person game $v$, i.e., $v(S)=v(T)$ whenever $|S|=|T|$, is a PAW-game, since $v_{h}=v_{h}^{i}$ for all $i \in N$, all $1 \leq h \leq n-1$. For another type of PAW-games see Example 3.5. According to the next proposition, a PAW-game can also be characterized in terms of the consistency of a certain system of quadratic equations.

Proposition 3.2. Let $n \geq 3$. A game $v \in G_{0}^{n}$ is a $P A W$-game if and only if there exist numbers $\bar{c}_{h} \in \mathbb{R}, h \in\{2,3, \ldots, n-1\}$, and numbers $d^{i}, i \in N$, such that $\bar{c}_{n-1} \neq 0$ and

$v_{h}-v_{h}^{i}=\bar{c}_{h} d^{i}$ for all $i \in N$, all $2 \leq h \leq n-1$.

Proof. Suppose that $v \in G_{0}^{n}$ is a PAW-game, i.e., (3.1) holds. Then, (3.2) also holds by choosing $d^{i}=v_{n-1}-v_{n-1}^{i}$ for all $i \in N, \bar{c}_{n-1}=1$ and $\bar{c}_{h}=c_{h}$ for all $2 \leq h \leq n-2$. To prove the converse implication, suppose that (3.2) holds. In particular, we have $v_{n-1}-v_{n-1}^{i}=\bar{c}_{n-1} d^{i}$ for all $i \in N$, where $\bar{c}_{n-1} \neq 0$. Now, it follows that

$v_{h}-v_{h}^{i}=\left(\bar{c}_{n-1}\right)^{-1} \bar{c}_{h}\left(v_{n-1}-v_{n-1}^{i}\right) \quad$ for all $i \in N$, all $2 \leq h \leq n-1$.

So, (3.2) implies (3.1); this establishes the equivalence of (3.1) and (3.2).

Let us give some remarks about this characterization of a zero-normalized $n$-person PAW-game. The system (3.2) has $n(n-2)$ quadratic equations in the $2 n-2$ unknowns $\bar{c}_{h}$, $2 \leq h \leq n-1$, and $d^{i}, i \in N$, whereas the left hand sides of (3.2) are determined by the data of the game $v$. Consider the quadratic subsystem of (3.2) for a fixed coalition size $h$, namely $v_{h}-v_{h}^{i}=\bar{c}_{h} d^{i}$ for all $i \in N$. In case that all left hand sides are zero, we take $\bar{c}_{h}=0$ and the unknowns $d^{i}, i \in N$, remain arbitrary. In case that there are nonzero left hand sides, we should have $\bar{c}_{h} \neq 0$ and the unknowns $d^{i}, i \in N$, are determined by $d^{i}=\left(\bar{c}_{h}\right)^{-1}\left(v_{h}-v_{h}^{i}\right)$ for all $i \in N$, where $\bar{c}_{h}$ remains arbitrary. Particularly, each such subsystem of (3.2) should be consistent with the special subsystem of (3.2) given by $d^{i}=\left(\bar{c}_{n-1}\right)^{-1}\left(v_{n-1}-v_{n-1}^{i}\right)$ for all $i \in N$. In other words, the consistency of two such subsystems requires that the corresponding ratio $\bar{c}_{h}\left(\bar{c}_{n-1}\right)^{-1}$ is uniquely determined by the ratios $\left(v_{h}-v_{h}^{i}\right)\left(v_{n-1}-v_{n-1}^{i}\right)^{-1}, i \in N$, provided that the denominator is not zero. If the consistency is met, then one of the numbers $\bar{c}_{h}, 2 \leq h \leq n-1$, is usually undetermined and it is standard to put $\bar{c}_{n-1}=1$.

An important observation is that the collinearity property with respect to $S h, E N S C$ and $C I S$ may hold although the game is not a PAW-game. Indeed, for the game $v$ of Example 2.5 conditions (3.1) can not be met because $v_{4}-v_{4}^{3}=0$ and $v_{3}-v_{3}^{3}=\frac{1}{10} \neq 0$, hence this game is not a PAWgame, but the three above-mentioned values are collinear.

Note that if a PAW-game $v \in G_{0}^{n}$ satisfies $v_{n-1}-v_{n-1}^{i}=0$ for all $i \in N$, or equivalently $\operatorname{ENSC}(v)=C I S(v)$, then it fol- lows from (3.1) that $v_{h}-v_{h}^{i}=0$ for all $i \in N$, all $2 \leq h \leq n-1$ and so, the Shapley value payoff to any player $i \in N$ reduces to $S h_{i}(v)=n^{-1} v_{n}=E N S C_{i}(v)=C I S_{i}(v)$. This coincidence of the three solution concepts with the egalitarian division rule of the overall profits is interpreted as a case of degenerated collinearity; in fact, we have also $E N A C(v)=C I S(v)$. Now, we shall give the interesting properties of the PAWgames which motivated us in introducing and studying this class of games.

Theorem 3.3. Let $v \in G_{0}^{n}$ be a PAW-game such that conditions (3.1) hold. We have

$$
S h_{i}(v)=n^{-1} v_{n}+\left[\sum_{h=2}^{n-1} h^{-1} c_{h}\right]\left(v_{n-1}-v_{n-1}^{i}\right)
$$

for all $i \in N$

(ii) The collinearity property holds with respect to Sh, ENSC and CIS, i.e.,

$\operatorname{Sh}(v)=\rho \operatorname{ENSC}(v)+(1-\rho) \operatorname{CIS}(v)$, where $\rho=\sum_{h=2}^{n-1} h^{-1} c_{h}$

(iii) Let $n \geq 4$ and $\operatorname{ENAC}(v) \neq C I S(v)$. Then, the collinearity property holds with respect to Sh, ENAC and CIS, i.e., $S h(v)=\gamma \operatorname{ENAC}(v)+(1-\gamma) \operatorname{CIS}(v)$, where $\gamma=\frac{(n-2) \rho}{(n-1) c_{n-2}}$

Proof. From formulas (2.13)-(2.15) and conditions (3.1) we derive that for all $i \in N$

$$
\begin{aligned}
S h_{i}(v) & =n^{-1} v_{n}+\sum_{h=2}^{n-1} h^{-1}\left(v_{h}-v_{h}^{i}\right) \\
& =n^{-1} v_{n}+\left[\sum_{h=2}^{n-1} h^{-1} c_{h}\right]\left(v_{n-1}-v_{n-1}^{i}\right) \\
& ={ } S_{i}(v)+\rho\left[\operatorname{ENSC}_{i}(v)-C_{1} S_{i}(v)\right] \\
& =\rho \operatorname{ENSC}_{i}(v)+(1-\rho) \operatorname{CIS}_{i}(v) .
\end{aligned}
$$

This proves parts (i) and (ii). In order to prove part (iii), if $E N A C_{j}(v) \neq C I S_{j}(v)$ for some $j \in N$, then (2.16) implies $v_{n-2}-v_{n-2}^{j} \neq 0$ and thus, $c_{n-2} \neq 0$ by applying (3.1) for $h=n-2$. Therefore, we have

$$
\begin{gathered}
\operatorname{ENSC}_{i}(v)-\operatorname{CSI}_{i}(v)=v_{n-1}-v_{n-1}^{i}=\left(c_{n-2}\right)^{-1}\left(v_{n-2}-v_{n-2}^{i}\right) \\
=\left[(n-1) c_{n-2}\right]^{-1}(n-2)\left[E N A C_{i}(v)-C I S_{i}(v)\right]
\end{gathered}
$$

for all $i \in N$. From this and part (ii), we conclude

$$
\begin{aligned}
S h(v)= & C I S(v)+\rho[\operatorname{ENSC}(v)-C I S(v)] \\
= & C I S(v)+\left[(n-1) c_{n-2}\right]^{-1}(n-2) \\
& \cdot \rho[\operatorname{ENAC}(v)-C I S(v)] \\
= & C I S(v)+\gamma[\operatorname{ENAC}(v)-C I S(v)] \\
= & \gamma \operatorname{ENAC}(v)+(1-\gamma) \operatorname{CIS}(v) .
\end{aligned}
$$

For a 4-person game the converse of part (ii) holds, as shown by:

Proposition 3.4. Let $v \in G_{0}^{4}$. Then, $v$ is a PAW-game if and only if the collinearity property holds with respect to $S h$, ENSC and CIS. 
Proof. The collinearity property holds if and only if for some number $\delta$ we have $S h(v)=C I S(v)+\delta[\operatorname{ENSC}(v)-$ $C I S(v)]$; if $n=4$, then this condition could be translated as $\frac{1}{3}\left(v_{3}-v_{3}^{i}\right)+\frac{1}{2}\left(v_{2}-v_{2}^{i}\right)=\delta\left(v_{3}-v_{3}^{i}\right) \quad$ for all $i \in N$, some $\delta \in \mathbb{R}$, or equivalently,

$v_{2}-v_{2}^{i}=2\left(\delta-\frac{1}{3}\right)\left(v_{3}-v_{3}^{i}\right) \quad$ for all $i \in N$, some $\delta \in \mathbb{R}$.

The latter statement is fully equivalent to conditions (3.1).

Note that such a result for games with more than four players is not true, as illustrated by Example 2.5, where we presented a 5-person game with the collinearity property which was not a PAW-game.

Now, we shall show that all unanimity games which are zero-normalized are PAW-games. Obviously, these games form a basis for the linear space $G_{0}^{n}$ of zero-normalized $n$ person games; however, not every game in $G_{0}^{n}$ is a PAWgame, because the class of zero-normalized PAW-games is not closed under addition, i.e., the sum of two $n$-person PAW-games is not necessarily a PAW-game.

Example 3.5. For any $T \subset N, T \neq \emptyset$, the unanimity n-person game $u_{T}$ is given by $u_{T}(S)=1$ if $S \supset T$ and $u_{T}(S)=0$, otherwise. Fix $T \subset N, T \neq \emptyset$, and write $t=|T|$. If $T=N$, then $u_{T}(S)=0$ for all $S \neq N, u_{T}(N)=1$, so that $\left(u_{T}\right)_{h}=\left(u_{T}\right)_{h}^{i}=0$ for all $i \in N$, all $1 \leq h \leq n-1$; hence, the unanimity game $u_{N}$ is a PAW-game, because (3.1) are satisfied. Suppose $T \neq N$. For any coalition size $h, 1 \leq h \leq n$, we get

$$
\left(u_{T}\right)_{h}=\left(\begin{array}{l}
n \\
h
\end{array}\right)^{-1} \sum_{S \in \Gamma_{h}} u_{T}(S)=\left(\begin{array}{l}
n \\
h
\end{array}\right)^{-1} \mid\left\{S \in \Gamma_{h} \mid T \subset S\right\},
$$

and a simple combinatorial computation gives

$$
\left(u_{T}\right)_{h}= \begin{cases}0 & \text { if } 1 \leq h<t, \\
\left(\begin{array}{l}
n \\
h
\end{array}\right)^{-1}\left(\begin{array}{l}
n-t \\
h-t
\end{array}\right) & \text { if } t \leq h \leq n .\end{cases}
$$

Similarly, for any $i \in N$ and any $1 \leq h \leq n-1$ we obtain

$$
\left(u_{T}\right)_{h}^{i}=\left(\begin{array}{c}
n-1 \\
h
\end{array}\right)^{-1} \sum_{S \in \Gamma_{h}^{i}} u_{h}(S)=\left(\begin{array}{c}
n-1 \\
h
\end{array}\right)^{-1}\left|\left\{S \in \Gamma_{h}^{i} \mid T \subset S\right\}\right|,
$$

and a simple combinatorial computation gives

$$
\begin{aligned}
& \left(u_{T}\right)_{h}^{i} \\
& =\left\{\begin{array}{cl}
0 & \text { if either } i \in T, 1 \leq h \leq n-1, \text { or } i \notin T, 1 \leq h<t, \\
\left(\begin{array}{c}
n-1 \\
h
\end{array}\right)^{-1}\left(\begin{array}{c}
n-1-t \\
h-t
\end{array}\right) & \text { if } i \notin T, t \leq h \leq n-1 .
\end{array}\right.
\end{aligned}
$$

From this we conclude that for any $i \in N$ and any $1 \leq h \leq n-1$, we have the results $\left(u_{T}\right)_{h}-\left(u_{T}\right){ }_{h}^{i}=0$ if $1 \leq h<t$ and

$$
\left(u_{T}\right)_{h}-\left(u_{T}\right)_{h}^{i}= \begin{cases}\left(\begin{array}{l}
n \\
h
\end{array}\right)^{-1}\left(\begin{array}{c}
n-t \\
h-t
\end{array}\right) & \text { if } i \in T, t \leq h \leq n-1, \\
\frac{-t}{n-t}\left(\begin{array}{c}
n \\
h
\end{array}\right)^{-1}\left(\begin{array}{c}
n-t \\
h-t
\end{array}\right) & \text { if } i \notin T, t \leq h \leq n-1 .\end{cases}
$$

We notice that if $2 \leq t \leq n-1$, then any corresponding unanimity game $u_{T}$ satisfies (3.2) with $\bar{c}_{h}=0$ for all $2 \leq h<t$,

$$
\begin{aligned}
& \bar{c}_{h}=\left(\begin{array}{l}
n \\
h
\end{array}\right)^{-1}\left(\begin{array}{l}
n-t \\
h-t
\end{array}\right) \frac{n}{n-t} \text { for all } t \leq h \leq n-1, \text { and } \\
& d^{i}=\frac{n-t}{n} \text { if } i \in T, \text { or } d^{i}=\frac{-t}{n} \text { if } i \notin T .
\end{aligned}
$$

Alternatively, it is straightforward to verify that conditions (3.1) hold in case $2 \leq t \leq n-1$ because we have for all $i \in N$

$$
\frac{\left(u_{T}\right)_{h}-\left(u_{T}\right)_{h}^{i}}{\left(u_{T}\right)_{n-1}-\left(u_{T}\right)_{n-1}^{i}}= \begin{cases}0 & \text { if } 1 \leq h<t, \\
\left(\begin{array}{c}
n-1 \\
t
\end{array}\right)^{-1}\left(\begin{array}{l}
h \\
t
\end{array}\right) & \text { if } t \leq h \leq n-1 .\end{cases}
$$

In particular, conditions (3.1) hold with $c_{h}=0$ for all $1 \leq h<t$, and $c_{h}=\left(\begin{array}{c}n-1 \\ t\end{array}\right)^{-1}\left(\begin{array}{l}h \\ t\end{array}\right)$ for all $t \leq h \leq n-1$, provided $2 \leq t \leq n-1$. We conclude that the zero-normalized unanimity games $u_{T}, T \subset N$, with $|T| \geq 2$ are PAW-games. According to Theorem 3.3 (ii), the collinearity property holds, that is $\operatorname{Sh}\left(u_{T}\right)=C I S\left(u_{T}\right)+\rho\left[E N S C\left(u_{T}\right)-C I S\left(u_{T}\right)\right]$, where $\rho \in \mathbb{R}$ is determined by

$\rho=\sum_{h=2}^{n-1} h^{-1} c_{h}=\left(\begin{array}{c}n-1 \\ t\end{array}\right)^{-1} \sum_{h=t}^{n-1} h^{-1}\left(\begin{array}{l}h \\ t\end{array}\right)=t^{-1}$.

The latter equality can be proved in an inductive manner by induction on $n$. Its proof is left to the reader.

Two other examples dealing with PAW-games can be found at the end of the next section (see Examples 4.4 and $4.6)$.

\section{Collinearity of values on the class of $k$-coalitional games}

Driessen and Funaki (1991) introduced the class of " $k$-coalitional games" and proved that under some additional condition, the collinearity between the Shapley value, the $E N S C$-value and the $C I S$-value holds for such games. The goal of this section is to investigate the relationship between the classes of $k$-coalitional games and PAW-games.

Definition 4.1. Let $k$ be an integer satisfying $1 \leq k \leq n-1$. An n-person game $v$ is called a $\boldsymbol{k}$-coalitional game if it satisfies the next two conditions:

(i) $v(S)=v(T)$ for all $S, T \subset N$ with $|S|=|T|<k$;

(ii) There exist numbers $\alpha_{T} \in \mathbb{R}$ for all $T \in \Gamma_{k}$ and numbers $\gamma_{k+1}, \gamma_{k+2}, \ldots, \gamma_{n-1} \in \mathbb{R}$ such that

$$
v(S)=\sum_{T \in \Gamma_{k} ; T \in S} \alpha_{T}+\gamma_{|S|}
$$

for all $S \subset N$ with $k+1 \leq|S| \leq n-1$.

This definition could be interpreted as follows. There are three types of coalitions in a $k$-coalitional game; essential coalitions, which consist of exactly $k$ players, small coalitions, having less than $k$ players, and large coalitions, with more than $k$ players and different from the grand coalition $N$. The numbers $\alpha_{T} \in \mathbb{R}$ represent the maximal profit obtainable from the formation of the essential coalition $T$ within any large coalition. Generally speaking, the worth of any large coalition described by (4.1) may be fully independent of the worth $v(T), T \subset S$, of essential coalitions. However, if it happens that the profit $\alpha_{T}$ of any essential coalition $T$ for its formation within a large coalition is the same as the worth $v(T)$ of the essential coalition in the game $v$, then the worth of any large coalition by (4.1) is fully determined by the worth of the essential subcoalitions and the constant corresponding to the size of the large coalition. The latter situation is found for example in any unanimity game $u_{R}, R \subset N, R \neq N$, $\emptyset$. If $|R|=k$, the unanim- 
ity game $u_{R}$ is a $k$-coalitional game, since the conditions (4.1) hold with $\alpha_{T}=u_{R}(T)$ for all $T \in \Gamma_{k}$ and $\gamma_{k+1}=\gamma_{k+2}=\ldots=$ $\gamma_{n-1}=0$ and obviously the symmetry conditions for small coalitions hold either.

Now, we treat a preliminary lemma involving $k$-coalitional games for the sake of comparison with PAW-games. We state the lemma for $k$-coalitional games with $1 \leq k \leq n-2$. First we mention that, in case $k=n-1$, we have $v_{h}=v_{h}^{i}$ for all $i \in N$, all $1 \leq h<n-1$, due to the symmetry, so the Shapley value reduces to $S h_{i}(v)=n^{-1} v_{n}+(n-1)^{-1}$. $\left(v_{n-1}-v_{n-1}^{i}\right)$ for all $i \in N$. This will be a result agreeing with the last statement in the lemma.

Lemma 4.2. Let $v \in G_{0}^{n}$ be a k-coalitional game such that conditions (4.1) hold, where $1 \leq k \leq n-2$. Denote

$$
\alpha_{k}:=\left(\begin{array}{c}
n \\
k
\end{array}\right)^{-1} \sum_{T \in \Gamma_{k}} \alpha_{T} \text { and } \alpha_{k}^{i}:=\left(\begin{array}{c}
n-1 \\
k
\end{array}\right)^{-1} \sum_{T \in \Gamma_{k}^{i}} \alpha_{T}
$$

for all $i \in N$. Then, for all $i \in N$, we have

$$
v_{h}-v_{h}^{i}=\left(\begin{array}{l}
h \\
k
\end{array}\right)\left(\alpha_{k}-\alpha_{k}^{i}\right) \text { for all } k+1 \leq h \leq n-1
$$

(ii)

$$
v_{h}-v_{h}^{i}=\left(\begin{array}{l}
h \\
k
\end{array}\right)\left(\begin{array}{c}
n-1 \\
k
\end{array}\right)^{-1}\left(v_{n-1}-v_{n-1}^{i}\right)
$$

for all $k+1 \leq h \leq n-1$

$$
\begin{aligned}
\text { (iii) } S h_{i}(v)= & n^{-1} v_{n}+k^{-1}\left[1-\left(\begin{array}{c}
n-1 \\
k
\end{array}\right)^{-1}\right]\left(v_{n-1}-v_{n-1}^{i}\right) \\
& +k^{-1}\left(v_{k}-v_{k}^{i}\right) .
\end{aligned}
$$

Proof. (i) Consider a fixed coalition size $h$ satisfying $k+1 \leq h \leq n-1$. Summing up the relevant equalities in (4.1) over all coalitions of size $h$, we obtain

$$
\begin{aligned}
\sum_{S \in \Gamma_{h}} v(S) & =\sum_{S \in \Gamma_{h}}\left[\sum_{T \in \Gamma_{k} ; T \subset S} \alpha_{T}+\gamma_{h}\right] \\
& =\sum_{S \in \Gamma_{h}} \sum_{T \in \Gamma_{k} ; T \subset S} \alpha_{T}+\left(\begin{array}{l}
n \\
h
\end{array}\right) \gamma_{h} .
\end{aligned}
$$

For a given $T \in \Gamma_{k}$, the corresponding profit $\alpha_{T}$ occurs $\left(\begin{array}{l}n-k \\ h-k\end{array}\right)$ times in the double sum of the expression in the right hand side, so the equality reduces to

$$
\sum_{S \in \Gamma_{h}} v(S)=\left(\begin{array}{l}
n-k \\
h-k
\end{array}\right) \sum_{T \in \Gamma_{k}} \alpha_{T}+\left(\begin{array}{l}
n \\
h
\end{array}\right) \gamma_{h}, \text { or } v_{h}=\left(\begin{array}{l}
h \\
k
\end{array}\right) \alpha_{k}+\gamma_{h}
$$

Similarly, summing up the relevant equalities in (4.1) over all coalitions of size $h$ not containing a fixed player $i \in N$, we obtain

$$
\begin{aligned}
& \sum_{S \in \Gamma_{h}^{i}} v(S)=\left(\begin{array}{c}
n-1-k \\
h-k
\end{array}\right) \sum_{T \in \Gamma_{k}^{i}} \alpha_{T}+\left(\begin{array}{c}
n-1 \\
h
\end{array}\right) \gamma_{h}, \text { or } \\
& v_{h}^{i}=\left(\begin{array}{l}
h \\
k
\end{array}\right) \alpha_{k}^{i}+\gamma_{h} .
\end{aligned}
$$

By subtracting the two partial results, we conclude that $v_{h}-v_{h}^{i}=\left(\begin{array}{c}h \\ k\end{array}\right)\left(\alpha_{k}-\alpha_{k}^{i}\right)$ for all $i \in N$, all $k+1 \leq h \leq n-1$. This proves part (i). Part (ii) is a direct consequence of part (i), by noting that $v_{n-1}-v_{n-1}^{i}=\left(\begin{array}{c}n-1 \\ k\end{array}\right)\left(\alpha_{k}-\alpha_{k}^{i}\right)$ for all $i \in N$. (iii) Let $i \in N$. Recall that $v_{h}-v_{h}^{i}=0$ for all $i \in N$, all $1 \leq h<k$, due to the symmetry property up to size $k$. From this and part (ii), we derive that the Shapley value payoff to player $i$ reduces to

$$
\begin{aligned}
S h_{i}(v)= & n^{-1} v_{n}+\sum_{h=k+1}^{n-1} h^{-1}\left(v_{h}-v_{h}^{i}\right)+k^{-1}\left(v_{k}-v_{k}^{i}\right) \\
= & n^{-1} v_{n}+\left(\begin{array}{c}
n-1 \\
k
\end{array}\right)^{-1}\left(v_{n-1}-v_{n-1}^{i}\right) \sum_{h=k+1}^{n-1} h^{-1}\left(\begin{array}{l}
h \\
k
\end{array}\right) \\
& +k^{-1}\left(v_{k}-v_{k}^{i}\right) .
\end{aligned}
$$

It remains to prove that

$$
\begin{aligned}
& \sum_{h=k+1}^{n-1} h^{-1}\left(\begin{array}{l}
h \\
k
\end{array}\right)=k^{-1}\left[\left(\begin{array}{c}
n-1 \\
k
\end{array}\right)-1\right], \text { or } \\
& \sum_{h=k}^{n-1} h^{-1}\left(\begin{array}{l}
h \\
k
\end{array}\right)=k^{-1}\left(\begin{array}{c}
n-1 \\
k
\end{array}\right)
\end{aligned}
$$

but this is a formula which can easily be proved by induction on $n(n \geq k+1)$, so that it has been left to the reader at the end of the previous section.

Note that the results of Lemma 4.2 hold also for $k=n-1$, as it can be seen by comparing the statement with the remark preceeding the lemma. Now, the lemma is the main tool in proving the central result.

Theorem 4.3. Let $v \in G_{0}^{n}$, and $n \geq 3$.

(i) Suppose that $v$ is 1 -coalitional. Then, $v$ is a PAW-game and the collinearity property holds, namely

$\operatorname{Sh}(v)=(n-1)^{-1}(n-2) \operatorname{ENSC}(v)+(n-1)^{-1} \operatorname{CIS}(v)$.

Moreover, ENAC $(v)=E N S C(v)$ whenever $n \geq 4$.

(ii) Suppose that v is $(n-1)$-coalitional. Then, $v$ is a PAWgame and the collinearity property holds, namely

$S h(v)=(n-1)^{-1} \operatorname{ENSC}(v)+(n-1)^{-1}(n-2) \operatorname{CIS}(v)$.

Moreover, ENAC $(v)=C I S(v)$.

(iii) Suppose that $v$ is $k$-coalitional, where $2 \leq k \leq n-2$. Then, the following three statements are equivalent:

(a) v is a PAW-game;

(b) there exists a number $c_{k} \in \mathbb{R}$ such that

$v_{k}-v_{k}^{i}=c_{k}\left(v_{n-1}-v_{n-1}^{i}\right)$ for all $i \in N$;

(c) the collinearity property holds, namely $S h(v)=$ $\rho \operatorname{ENSC}(v)+(1-\rho) \operatorname{CIS}(v)$ with $\rho=k^{-1}\left[1+c_{k}-\left(\begin{array}{c}n-1 \\ k\end{array}\right)^{-1}\right]$, where $c_{k}$ is the same number as above.

Proof. (i) Lemma 4.2(ii) applied for $k=1$ yields $v_{h}-v_{h}^{i}=(n-1)^{-1} h\left(v_{n-1}-v_{n-1}^{i}\right)$ for all $i \in N$, all $2 \leq h \leq n-1$. Thus, conditions (3.1) hold with $c_{h}=(n-1)^{-1} h$ for all $2 \leq h \leq n-1$. Hence, $v$ is a PAW-game and the collinearity property follows from Theorem 3.3 (ii), where the coefficient $\rho$ is computed by

$\rho=\sum_{h=2}^{n-1} h^{-1} c_{h}=\sum_{h=2}^{n-1}(n-1)^{-1}=(n-1)^{-1}(n-2)$.

Note that if $n \geq 4$, then $E N A C_{i}(v)-C I S_{i}(v)=(n-2)^{-1}(n-1)$ $\left(v_{n-2}-v_{n-2}^{i}\right)=v_{n-1}-v_{n-1}^{i}=E N S C_{i}(v)-C I S_{i}(v)$ for all $i \in N$. So, $E N A C(v)=E N S C(v)$ if $n \geq 4$. 
(ii) Obviously, (3.1) holds for an $(n-1)$-coalitional game by choosing $c_{n-1}=1$ and $c_{h}=0$ for all $2 \leq h \leq n-2$. Thus, $v$ is a PAW-game and the collinearity property holds. Note that $\operatorname{ENAC}_{i}(v)-\operatorname{CIS}_{i}(v)=(n-2)^{-1}(n-1)\left(v_{n-2}-v_{n-2}^{i}\right)=0$ for all $i \in N$ and so, $\operatorname{ENAC}(v)=C I S(v)$.

(iii) In view of Lemma 4.2 (ii), we notice that (3.1) holds for all $2 \leq h \leq n-1, h \neq k$, by choosing $c_{h}=0$ for all $2 \leq h<k$, and $c_{k}=\left(\begin{array}{c}h \\ k\end{array}\right)\left(\begin{array}{c}n-1 \\ k\end{array}\right)-1$ for all $k+1 \leq h \leq n-1$. From this it follows immediately that (3.1) and (4.3) are equivalent, so that the first two statements are equivalent. If (4.3) holds, then formula (4.2) for the Shapley value reduces to

$$
\begin{aligned}
S h_{i}(v) & =n^{-1} v_{n}+k^{-1}\left[1+c_{k}-\left(\begin{array}{c}
n-1 \\
k
\end{array}\right)^{-1}\right]\left(v_{n-1}-v_{n-1}^{i}\right) \\
& =\operatorname{CIS}_{i}(v)+\rho\left[\operatorname{ENSC}_{i}(v)-\operatorname{CIS}_{i}(v)\right] \text { for all } i \in N
\end{aligned}
$$

therefore, (4.3) implies the collinearity property. To prove the converse implication, assume that the collinearity property holds, i.e., there exists a number $\bar{\rho} \in \mathbb{R}$ such that $S h(v)=C I S(v)+\bar{\rho}[\operatorname{ENSC}(v)-C I S(v)]$. We have $S h_{i}(v)=$ $n^{-1} v_{n}+\bar{\rho}\left(v_{n-1}-v_{n-1}^{l}\right)$ for all $i \in N$, which together with formula (4.2) yields that for all $i \in N$

$$
\begin{aligned}
\bar{\rho}\left(v_{n-1}-v_{n-1}^{i}\right) & =k^{-1}\left[1-\left(\begin{array}{c}
n-1 \\
k
\end{array}\right)^{-1}\right]\left(v_{n-1}-v_{n-1}^{i}\right) \\
& +k^{-1}\left(v_{k}-v_{k}^{i}\right)
\end{aligned}
$$

or equivalently

$$
v_{k}-v_{k}^{i}=\left[k \bar{\rho}-1+\left(\begin{array}{c}
n-1 \\
k
\end{array}\right)^{-1}\right]\left(v_{n-1}-v_{n-1}^{i}\right) \text { for all } i \in N \text {. }
$$

Thus, (4.3) holds by choosing $c_{k}=k \bar{\rho}-1+\left(\begin{array}{c}n-1 \\ k\end{array}\right)^{-1}$, provided that $\bar{\rho}$ is determined by the collinearity property. This completes the proof.

Theorem 4.3 says that all zero-normalized $k$-coalitional games $v$ for which $S h(v), E N S C(v)$ and CIS $(v)$ are collinear payoff vectors, are $\mathrm{PAW}$-games. The corresponding formulas involving the collinearity of the three vectors were proved by Driessen and Funaki (1991) using different methods (see Theorems 6.3,6.6 and 6.9 there). The reader may verify that condition (4.3) is equivalent to the corresponding condition of Theorem 6.9 in [4]. We emphasize that the result of Theorem 4.3 (iii) above is more precise than the result of Theorem 6.9 in [4], due to the fact that condition (4.3) is not only sufficient but also necessary for the collinearity property of zero-normalized $k$ coalitional games with $2 \leq k \leq n-2$.

Now, we present an example of a PAW-game which is not $k$-coalitional for any integer $k, 1 \leq k \leq n-1$. As any PAWgame has the collinearity property, a fact shown by Theorem 3.3, this means that by introducing the PAW-games we have enlarged the class of games for which it is known that the Shapley value is easily computable due to the collinearity. The example shows also an easy way of using Lemma 4.2 for proving that a game is not $k$-coalitional.

Example 4.4. Consider the zero-normalized 5-person game $v$ given by
$v(S)=\left\{\begin{array}{l}0 \text { if } 1 \notin S, \\ 1 \text { if } 1 \in S, \text { and }|S|=2 \text {, or } 4, \\ 2 \text { if } 1 \in S, \text { and }|S|=3 \text {, or } 5 .\end{array}\right.$

Straightforward calculations yield all average worth as well as all differences of average worth:

$$
\begin{aligned}
& v_{2}=\frac{4}{10}, v_{2}^{1}=0, v_{2}^{i}=\frac{3}{6}, v_{2}-v_{2}^{1}=\frac{2}{5}, v_{2}-v_{2}^{i}=\frac{-1}{10}, \\
& v_{3}=\frac{12}{10}, v_{3}^{1}=0, v_{3}^{i}=\frac{6}{4}, v_{3}-v_{3}^{1}=\frac{6}{5}, v_{3}-v_{3}^{i}=\frac{-3}{10}, \\
& v_{4}=\frac{4}{5}, v_{4}^{1}=0, v_{4}^{i}=\frac{1}{1}, v_{4}-v_{4}^{1}=\frac{4}{5}, v_{4}-v_{4}^{i}=\frac{-1}{5},
\end{aligned}
$$

where $i \in\{2,3,4,5\}$. Obviously, conditions (3.1) hold by choosing $c_{2}=\frac{1}{2}, c_{3}=\frac{3}{2}, c_{4}=1$, hence $v$ is a PAW-game. Notice that Theorem 3.3 (ii) tells us that $S h(v)=E N S C(v)$, because $\rho=\frac{1}{2} c_{2}+\frac{1}{3} c_{3}+\frac{1}{4} c_{4}=1$. By easy computations we obtain

$\operatorname{CIS}(v)=\frac{2}{5}(1,1,1,1,1), \operatorname{ENSC}(v)=\frac{1}{5}(6,1,1,1,1)$, $\operatorname{ENAC}(v)=(2,0,0,0,0)$.

We get also $S h(v)=\frac{1}{2} \operatorname{ENAC}(v)+\frac{1}{2} C I S(v)$. Clearly, the game $v$ is neither 3-coalitional nor 4-coalitional because the symmetry property does not hold for 2-person coalitions. If the game $v$ would be $k$-coalitional with $k=1$ or $k=2$,

then by Lemma 4.2 (ii) we would have $\frac{3}{2}=\frac{v_{3}-v_{3}^{i}}{v_{4}-v_{4}^{i}}=\frac{\left(\begin{array}{l}3 \\ k\end{array}\right)}{\left(\begin{array}{l}4 \\ k\end{array}\right)}$ for all $i \in N$. These equalities do not hold for $k=1$ or $k=2$, hence our PAW-game is not $k$-coalitional for any $1 \leq k \leq 4$.

Note also another use of Theorem 4.3 (iii) shown by the following:

Example 4.5. Consider once again the zero-normalized 4-person game $v$ of Example 2.4. This game is a 2-coalitional game with respect to the profits $\alpha_{12}=0, \alpha_{13}=\alpha_{23}=$ $\alpha_{34}=\frac{1}{2}, \alpha_{14}=\alpha_{24}=1$ and the constant $\gamma_{3}=0$. Notice that $v$ is not a PAW-game because there exists no $c_{2} \in \mathbb{R}$ such that $v_{2}-v_{2}^{i}=c_{2}\left(v_{3}-v_{3}^{i}\right)$ for all $i \in N$. In view of Theorem 4.3 (iii), this fact fully explains why there is no collinearity of the payoff vectors $S h(v), E N S C(v)$ and $C I S(v)$.

Example 4.6. Let player 1 be a landowner and players $2,3, \ldots, m+1$ landless peasants of the same type who have nothing to contribute but their labour in order to cultivate the land. Let $f:\{0,1, \ldots, m\} \rightarrow \mathbb{R}$ be a production function, where $f(t)$ represents the monetary value of the crop of the land cultivated by $t$ peasants, $0 \leq t \leq m$, who are hired by the landowner. It is supposed that the landowner by himself can not produce anything, i.e., $f(0)=0$, and that $f$ is a nondecreasing function, i.e., $f(t+1) \geq f(t)$ for all $0 \leq t \leq m-1$. This economic situation can be modelled as a cooperative $(m+1)$-person game $v$ with player set $N=$ $\{1,2,3, \ldots, m+1\}, m \geq 1$, whose characteristic function $v: 2^{N} \rightarrow \mathbb{R}$ is given by

$v(S)=0 \quad$ if $1 \notin S$, and $v(S)=f(|S|-1)$ if $1 \in S$.

Thus, the worth of any coalition containing the landowner equals the monetary output produced by the peasants in the coalition. Any coalition consisting of peasants only has no worth, since the peasants do not own any land. Notice 
that this landlord game $v$ is zero-normalized, i.e., $v(\{i\})=0$ for all $i \in N$. Our purpose is to prove that landlord games are PAW-games. In view of (2.7) and (2.8), all average worth are

$$
\begin{aligned}
v_{h}=\left(\begin{array}{c}
m+1 \\
h
\end{array}\right)^{-1}\left(\begin{array}{c}
m \\
h-1
\end{array}\right) f(h-1)=\frac{h}{m+1} & f(h-1) \\
& \text { for all } 1 \leq h \leq m+1 ;
\end{aligned}
$$$$
v_{h}^{i}=\left(\begin{array}{c}
m \\
h
\end{array}\right)^{-1}\left(\begin{array}{c}
m-1 \\
h-1
\end{array}\right) f(h-1)=\frac{h}{m} f(h-1), \text { and } v_{h}^{1}=0
$$

for all $1 \leq h \leq m$, all $i \in N \backslash\{1\}$. Consequently, we have $v_{h}-v_{h}^{1}=\frac{h}{m+1} f(h-1)$ and $v_{h}-v_{h}^{i}=\frac{-h}{m(m+1)} f(h-1)$

for all $1 \leq h \leq m$, all $i \in N \backslash\{1\}$. Thus, conditions (3.1) hold with $c_{h}=\frac{h f(h-1)}{m f(m-1)}$ for all $2 \leq h \leq m$, provided that $f(m-1) \neq 0$ (otherwise, choose $c_{h}=0$ for all $2 \leq h \leq m$ ). Hence, the landlord game $v$ is a PAW-game and due to Theorem 3.3 (ii), the collinearity property holds, that is $S h$ $(v)=\rho \operatorname{ENSC}(v)+(1-\rho) \operatorname{CIS}(v)$, where the coefficient $\rho$ is computed by

$$
\rho=\sum_{h=2}^{m} h^{-1} c_{h}=\sum_{h=2}^{m} \frac{f(h-1)}{m f(m-1)}=[m f(m-1)]^{-1} \sum_{t=1}^{m-1} f(t)
$$

provided that $f(m-1) \neq 0$. Obviously, (2.13) yields

$$
\begin{aligned}
& S h_{1}(v)=(m+1)^{-1} \sum_{t=0}^{m} f(t) \text { and } \\
& S h_{i}(v)=(m+1)^{-1}\left[f(m)-m^{-1} \sum_{t=1}^{m-1} f(t)\right]
\end{aligned}
$$

for all $i \in N \backslash\{1\}$. Generally speaking, the landlord game $v$ is not $k$-coalitional for any $3 \leq k \leq m$, because the symmetry property does not hold for 2-person coalitions. If the game $v$ would be $k$-coalitional with $k=1$, or $k=2$, then by Lemma 4.2 (ii) we would have

$$
\frac{h f(h-1)}{(h+1) f(h)}=\frac{v_{h}-v_{h}^{i}}{v_{h+1}-v_{h+1}^{i}}=\frac{\left(\begin{array}{l}
h \\
k
\end{array}\right)}{\left(\begin{array}{c}
h+1 \\
k
\end{array}\right)}=\frac{h+1-k}{h+1}
$$

for all $i \in N$, all $k+1 \leq h \leq m-1$. So, 1-coalitionality would require $f(h-1)=f(h)$ for all $2 \leq h \leq m-1$. Without going into technical details, it appears that $v$ is 1-coalitional iff $f(1)=f(2)=\cdots=f(m-1)$.

\section{References}

1. Dragan I (1992) An average per capita formula for the Shapley value. Libertas Math 12:139-146

2. Driessen TSH (1985) Properties of 1-convex n-person games. OR Spektrum 7:19-26

3. Driessen TSH (1988) Cooperative Games, Solutions, and Applications. Kluwer Academic Publishers, Dordrecht

4. Driessen TSH, Funaki Y (1991) Coincidence of and collinearity between game theoretic solutions. OR Spektrum 13:15-30

5. Driessen TSH, Funaki Y (1993 a) The egalitarian non-average contribution method for cooperative games. Memorandum No. 1135, Department of Applied Mathematics, University of Twente, Enschede, The Netherlands

6. Driessen TSH, Funaki Y (1993b) Reduced game properties of egalitarian division rules for cooperative games. Memorandum No. 1136, Department of Applied Mathematics, University of Twente, Enschede, The Netherlands

7. Funaki Y (1986) Upper and lower bounds of the kernel and nucleolus. Int J Game Theory 15:121-129

8. Gambarelli G, Zambruno G (1979) Analytical reformulation of the Shapley value of a game. In: Giappicheli G (ed) Atti del Primo Convegno, A.M.A.S.E.S., Torino, pp 197-211

9. Legros P (1986) Allocating joint costs by means of the nucleolus. Int J Game Theory 15:109-119

10. Maschler M (1982) The worth of a cooperative enterprise to each member. In: Games, Economics and Time Series Analysis. Physica, Wien, pp 67-73

11. Moulin H (1985) The separability axiom and equal-sharing methods. J Econ Theory 36: 120-148

12. Peleg B (1992) Voting by count and account. In: Selten R (ed) Rational Interaction. Essays in honor of John C. Harsanyi. Springer, Berlin Heidelberg, pp 45-51

13. Shapley LS (1953) A value for n-person games. In: Kuhn H, Tucker AW (eds) Contributions to the Theory of Games II. Ann. Math. Studies Vol 28, Princeton University Press, Princeton, New Jersey, pp 307-317

14. Young HP, Okada N, Hashimoto T (1982) Cost allocation in water resources development. Water Resourc Res 18:463-475 